\title{
The distance and space distribution of bulge globular clusters
}

\author{
Sergio Ortolani, ${ }^{1}$ Beatriz Barbuy ${ }^{2}$ and Eduardo Bica ${ }^{3}$ \\ ${ }^{1}$ Dipartimento di Astronomia, University of Padova, \\ Vicolo dell'Osservatorio 2, 35122, Padova, Italy \\ email: sergio.ortolani@unipd.it \\ ${ }^{2}$ Universidade de São Paulo, IAG, Rua do Matao 1226, Cidade Universitaria, \\ São Paulo 05508-900, Brazil \\ email: barbuy@astro.iag.usp.br \\ ${ }^{3}$ Universidade Federal do Rio Grande do Sul, Departamento de Astronomia CP 15051, RS, \\ Porto Alegre 91501-970, Brazil \\ email: bica@if.ufrgs.br
}

\begin{abstract}
We present the distribution of Galactic bulge globular clusters and a method based on simultaneous detection of field and cluster horizontal branches to derive the cluster distances. This method has the advantage of being independent of both reddening and the reddening law, $R_{V}=A_{V} / E(B-V)$. The vast majority of clusters projected in the direction of the Galactic bulge are located on the near side of the Galactic Center. Deviations from the reddening law do not seem to be responsible for this peculiarity. We need to introduce a peculiar, steep dependence of the absolute horizontal-branch magnitude with metallicity in the metal-rich regime if we want to reproduce a symmetrical distribution. Instead, if the observed distribution is correct, we expect a rather large number of bulge globular clusters are still to be discovered.
\end{abstract}

Keywords. Galaxy: bulge, globular clusters: general, Galaxy: structure

\section{Introduction}

The Galactic globular clusters are strongly concentrated towards the Galactic Center. In a $20^{\circ} \times 20^{\circ}$ field, there are about 74 known globular clusters, of which 60 are located closer than $4 \mathrm{kpc}$ (in projection) from the Galactic Center. They represent almost half of the total number of globular clusters known in the entire Galaxy. A recent review describes the properties of the globular clusters located in a $5^{\circ} \times 5^{\circ}$ field (Barbuy et al. 1998), showing that the vast majority, possibly all, belong to the family of bulge clusters. Now, more than 10 years since their discovery, we may have uncertain distances because of the high reddening and the relatively poorly known absolute magnitude of the horizontal branch (HB), which is currently used as standard candle for most of the clusters. Here, we present a detailed overview of a larger field $\left(6^{\circ} \times 6^{\circ}\right)$ containing 23 clusters, and investigate their distance distribution, apply checks using different methods, and discuss possible biases and the consequences of the most common assumptions.

\section{Overview}

The distances to the clusters in the present study are based on the HB magnitudemetallicity relation, $M_{V}(\mathrm{HB})=0.16[\mathrm{Fe} / \mathrm{H}]+0.98$. The $\mathrm{HB}$ visual magnitudes and reddening values are taken from individual published studies. The results show that all but two clusters are located on the near side of the Galactic Center (assuming a conservative distance to the Galactic Center of $8 \mathrm{kpc}$ ), and at an average distance of about $6.3 \mathrm{kpc}$ from the Sun (see Figure 1). 


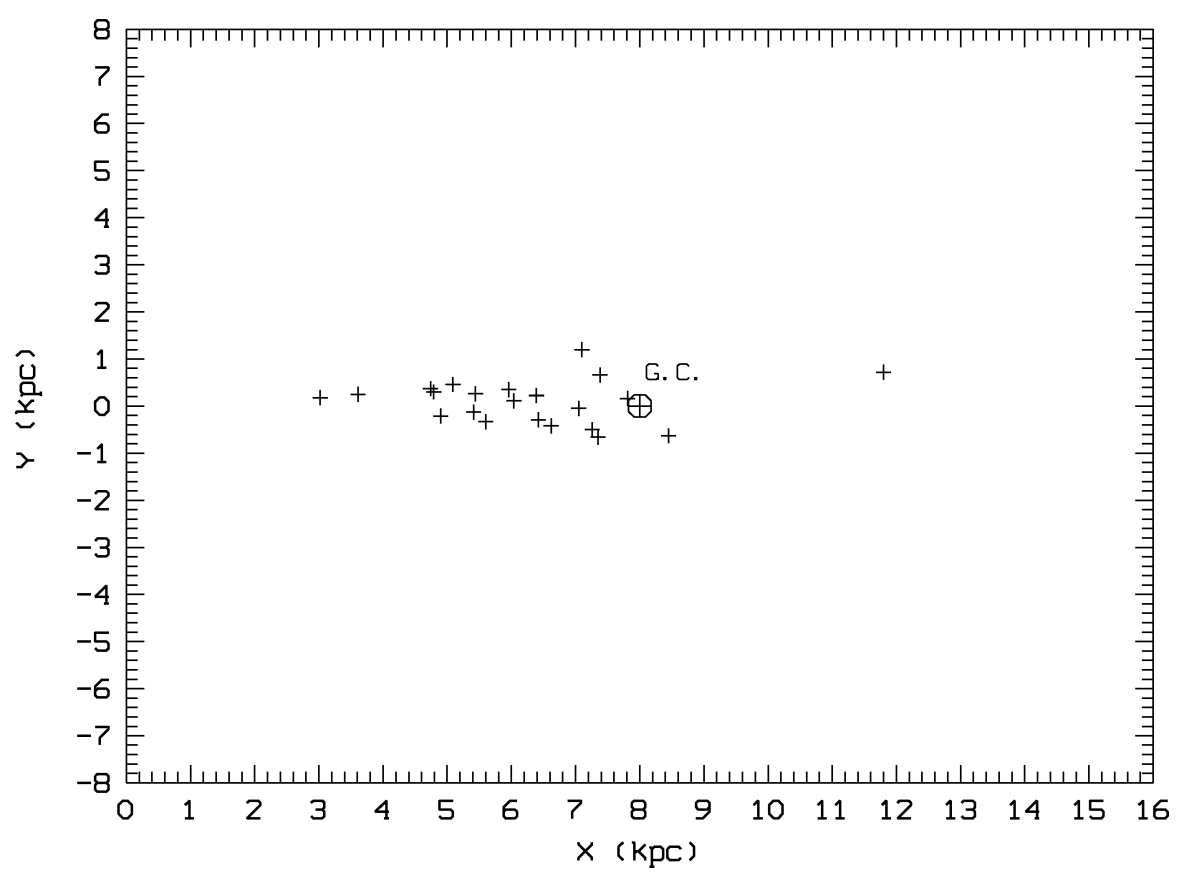

Figure 1. Distribution of the globular clusters in a $6^{\circ} \times 6^{\circ}$ field projected onto the Galactic plane.

Considering that there is no trend of distance with reddening (Barbuy et al. 1998), we checked the relation between visual magnitude and reddening to see if the slope of the reddening law might be significantly different from the standard $R_{V}=A_{V} / E(B-V)=3.1$. As expected from the long line of sight through the Galactic disk, the average value of 3.1 seems a reasonable interpolation of the data. If we exclude that this choice of reddening law is responsible for the asymmetrical distribution of the globular clusters around the Galactic Center, we need to check the assumed relation for the absolute magnitude of the HB. A small change in the coefficient or in the zeropoint (0.1$0.2 \mathrm{mag}$ ) does not change the results significantly. For an average distance of $8 \mathrm{kpc}$, one must impose a zeropoint differing by about 0.6 mag from the assumed value, which is not compatible with the distribution of the halo clusters. Another alternative could be a higher luminosity dependence with metallicity, combined with a lower zeropoint, to match the zeropoint for the lower-metallicity halo clusters. A coefficient as high as about 0.5 would be required, still incompatible with the much lower coefficients derived from halo clusters and field stars. It is clear that an independent check of the distances is needed. For this purpose, we adopted a different approach, based on the simultaneous detection of stellar field and cluster HBs in the same frame, i.e., affected by the same reddening. We assume that the field HB (appearing as a red clump) corresponds to the bulge field $\mathrm{HB}$ and that the barycentric position of the bulge field coincides with the Galactic Center.

We determined the luminosity differences between the HBs of the clusters and the field for all clusters where the field HB was clearly defined in the same frame. In some cases, we used proper-motion separation of the two components (notably for NGC 6553 and NGC 6528). This method is independent of both the reddening and the reddening law adopted. The results from a subsample of 12 clusters indicate that the cluster HBs are systematically brighter than the field by an average of $0.31 \mathrm{mag}$ in the $V$ band. Only three 
clusters have a fainter HB than the corresponding field stars. On average, the magnitude difference decreases with our original distance measurements discussed above, indicating that the measured luminosity difference is the result of a real distance effect and not something peculiar.

If we interpret this result as purely an effect of distance differences between the baricentric position of the field population and the cluster positions, we again obtain a similar result to that already derived using the standard calibration and the individual reddening for each cluster. However, in doing so we ignore differential metallicity effects on the HB luminosity. From the literature, we derive that the sample clusters have an average metallicity of about $[\mathrm{Fe} / \mathrm{H}]=-0.9$. The bulge field has a metallicity around solar, or somewhat higher (Zoccali et al. 2008). It is easy to see that if we want to match the average cluster distance modulus with that of the field, we need a coefficient for the HB luminosity dependence on metallicity of about 0.4 between about $[\mathrm{Fe} / \mathrm{H}]=-1.3$ and solar metallicity. This is marginally compatible with the halo clusters, and only if we assume a sudden change in slope around a metallicity between $[\mathrm{Fe} / \mathrm{H}]=-1.3$ and -1.0 . A change of slope in this interval was proposed by McNamara (1999). An alternative is that the currently known sample of globular clusters is biased due to a peculiar combination of distance and reddening and that we are missing most of the clusters on the other side of the Galactic Center in a relatively narrow cone subtending at least 10-20 degrees.

\section{Implications}

Studies of the distances of Galactic bulge clusters show an anomalous distribution, with a concentration on the near side of the Galactic Center. This asymmetric distribution is apparently confirmed by the systematically brighter HBs of the clusters compared to the bulge field HBs projected in the same direction. To match their distances, we need a rather different calibration equation for the absolute magnitude of the $\mathrm{HB}$ of the bulge clusters from that representing the halo clusters. An alternative explanation is that we are missing most of the low-latitude clusters projected in the direction of the Galactic bulge due to reddening, distance issues and crowding effects. Future near-infrared surveys may reveal these clusters, provided that crowding is not a major problem.

\section{References}

Barbuy, B., Bica, E., \& Ortolani, S. 1998, A\&A, 333, 117

McNamara, D. H. 1999, PASP, 111, 489

Zoccali, E., Hill, V., Lecureur, A., Barbuy, B., Renzini, A., Minniti, D., Gomez, A., \& Ortolani, S. $2008, A \xi A, 486,177$ 